\title{
Comprehensive Approach To Rheumatic Fever and Rheumatic Heart Disease Prevention and Control: The Nepalese Model
}

\author{
Prakash Raj Regmi
}

National Academy of Medical Sciences, Kathmandu, Nepal Corresponding Author: Prakash Raj Regmi, National Academy of Medical Sciences, Bir Hospital, Mahaboudha, Kathmandu, Nepal Email:pregmi68@yahoo.com

\begin{abstract}
Rheumatic Heart Disease represents a huge public health burden in developing countries with significant morbidity and mortality. This disease is preventable and has been successfully controlled through the implementation of register-based control programs. The Nepal Heart Foundation, a non-governmental organization was the first to initiate community activities with focus on RHD prevention and control. This organization in 2007 was successful in launching the RHD prevention and control program in partnership with the government of Nepal. The Nepalese model of RHD control program is an example of a comprehensive, diagonal and register-based approach to RHD prevention and control in low resource settings. The early outcomes and impact of the program are encouraging. This paper provides an overview of the Nepalese model of RHD prevention and control program.
\end{abstract}

Keywords : Rheumatic Fever, Rheumatic Heart Disease, Rheumatic Heart Disease Control Program

\section{Introduction}

No child should die from a preventable disease. Rheumatic Fever (RF) and Rheumatic Heart Disease (RHD) are among those diseases which are preventable but has affected more than 15.6 million people worldwide causing 233000 deaths annually. They also cause significant morbidity in young people. ${ }^{1}$ The vast majority of cases of $\mathrm{RF}$ and RHD occur in developing countries, including Nepal.

RHD is a major pediatric heart problem in Nepal. It accounts for around 3000 premature deaths annually, 1.0 to 1.35 per 1000 school children of age 5-16 years suffer from this diseases. ${ }^{2,3,4,5}$ In a school based cross sectional study in eastern Nepal, the prevalence of RHD (including subclinical) was found to be 10 per 1000 school children. ${ }^{6}$ This study shows that subclinical RHD exist in large number in the community increasing the burden from RHD by several folds.

World Health Organization (WHO) led a global program of RHD control which was established in 16 countries and later expanded to 22 in the mid-1980s. ${ }^{7}$ Although underfunded this global program had some remarkable achievements: the concept of register based control programs was borne, as was the idea of screening school-aged children for RHD; the WHO published the first global guideline on RHD; and some pilot programs managed to persist and even later reported dramatic reductions in disease burden. ${ }^{8,9}$

In Nepal the burden of RF/RHD had remained unchanged for more than two decades till the establishment of National RF/RHD prevention and control program in 2007. The efforts made after implementation of the program resulted in start of decline in RHD burden. ${ }^{10}$

\section{A Conceptual Framework For Comprehensive RHD Control Programs}

The World Heart Federation (WHF) in 2013 published a position statement outlining the five key strategic targets required to meet its strategic goal for RHD: a $25 \%$ reduction in premature deaths from RF and RHD among individuals aged $<25$ years by the year 2025. ${ }^{11}$ A number of barriers have been identified that are currently preventing the control of RF and RHD. They are:

- Neglect of RF and RHD: there is a relative lack of engagement in disease control by governments, civil society, patient advocates and funding agencies.

- Scarcity of data and scientific knowledge: contemporary data is scare.

- Resources for research: there is a great need for resource allocation to conduct robust research.

- Vaccines: Group A Streptococcus (GAS) Vaccine is required for effective population-level primary prevention of RF.

- Access to health care: Barriers exist to optional primary, secondary and tertiary medical and surgical care

The primary aim of the position statement is to serve as a guide on how to achieve the objective to control RHD and eliminating RF. Five strategic targets have been identified:

1. Comprehensive register-based control programs.

2. Global access to Benzathine penicillin $\mathrm{G}$

3. Identification and development of public figures as RHD champions

4. Expansion of RHD training hubs

5. Support for vaccine development 
Moreover, this statement is intended to provide the foundation for governments, civil society, patient advocates, clinicians, researchers and funding agencies to develop partnership and unite global efforts to control RF and RHD.

$\mathrm{RF} / \mathrm{RHD}$ has been successfully controlled in a number of settings through the implementation of register-based control programs. ${ }^{12,13,14}$ The register based approach to RF/RHD control has been recommended by the WHO and WHF for many years. ${ }^{15,16,17}$ Most control programs are 'Comprehensive' in that they attempt to intervene at multiple points along the protracted etiological pathway of RF/RHD. ${ }^{18,19}$ In 2013 the recommended components of comprehensive RHD control programs were collated and structured into a conceptual framework. ${ }^{20}$ This framework provides a structure for Tools for Implementing control Programs (TIPs) which was published by WHF in 2014. TIPs provide a resource for people and places contemplating an RHD control Program. The collation of decades of implementation experience from around the world provides a solid foundation for customized program development. TIPs present a simple overview of RF, RHD and opportunities for intervention, alongside a priority based framework for program delivery. The resource is intended to support the description, development and delivery of RHD control programs. ${ }^{21}$

\section{What is a comprehensive RHD control program?}

There are many opportunities to intervene on the pathway from GAS to RHD. Traditionally these have been divided into primordial, Primary, Secondary and tertiary interventions (Fig 1)

RHD control program is considered 'Comprehensive' if it includes all the components of primary, secondary and tertiary prevention. The importance of this kind of comprehensive approach to RHD control is increasingly recognized. ${ }^{21}$

\section{National RF/RHD Prevention And Control Program In Nepal}

\section{The History}

Nepal Heart Foundation (NHF) a forerunner non-governmental organization established in 1988 initiated community activities with focus on RHD prevention through one of its five national programs with the name "Save the Children's Heart Program" in 1990. During those days RHD was among the leading causes of admission to cardiology services and Cardio thoracic surgery.2 Due to lack of surgical facilities for RHD, patients were forced to travel to India for heart surgery. Patients who could not afford had no options for treatment. This led to huge member of premature deaths from RHD. As a result of several years advocacy led by NHF, Shahid Gangalal National Heart Centre (SGNHC) was established and became functional in 1998. The government of Nepal (GoN) then started supporting for valve surgeries of RHD patients providing a good number of prosthetic valves free of cost.

The demand for free heart valves increased year by year and the waiting list crossed over one year. RHD prevention program was the need of time. By that time NHF developed a project proposal on RHD prevention and control and handed over to the Ministry of Health and Population (MoHP) in 2006. MoHP decided to launch National RF/RHD prevention and control program in 2006.The budget allocation was NRs. 3 million (USD 30000). NHF was then given the task of implementing the program in 2007.

\section{Program Design}

The NHF investigated a number of models for delivering disease-specific health care while developing the national program for control of RF and RHD. In particular, decisions

Figure 1: Opportunities for Intervention in RF and RHD

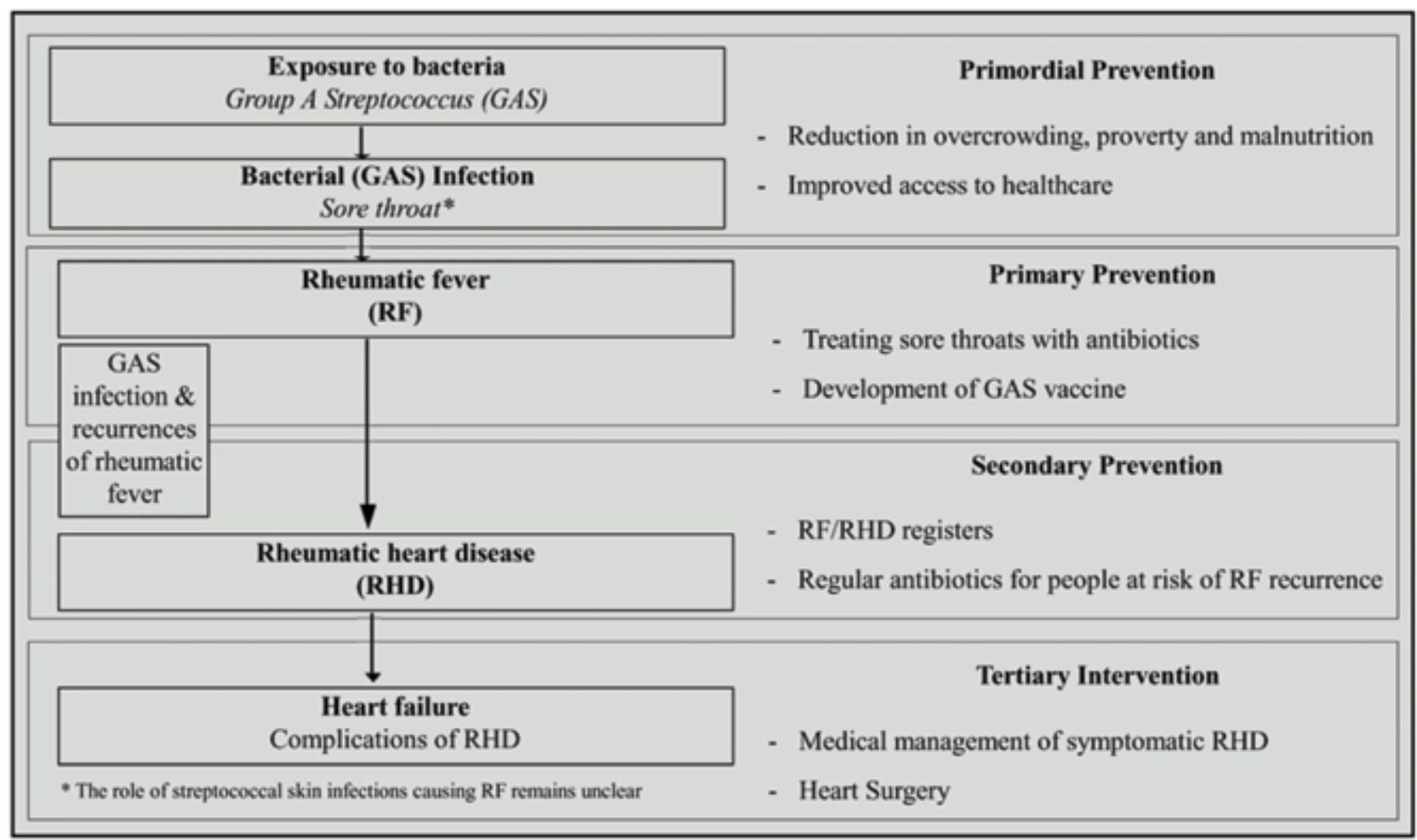


were required about the relative contribution of independent disease specific activities (vertical) and integration of RHD care delivery into the broader health system (horizontal). ${ }^{23}$ The NHF identified that a purely vertical approach was prohibitively costly and a purely horizontal approach lacked the urgent focus needed for reducing RF/RHD morbidity and mortality. A combination (diagonal approach) was chosen in order to focus on RHD within the framework of the existing health care system. The Nepalese RHD control program is a diagonal partnership between the GoN and the NHF.

The national RF/RHD prevention and control program has 3 objectives and 8 elements, outlined in table 1 and 2 . The overall

Table 1 : Core program objectives

\begin{tabular}{|r|l|}
\hline 1. & $\begin{array}{l}\text { Early detection and registration of } \\
\text { RF/RHD patients }\end{array}$ \\
\hline 2. & $\begin{array}{l}\text { Establishment of centers for safe } \\
\text { administration of Benzathine Penicillin G } \\
\text { (BPG) injection for secondary prophylaxis }\end{array}$ \\
\hline 3. & $\begin{array}{l}\text { Establishment of a national strategy for } \\
\text { RF/RHD prevention and control with } \\
\text { development of RHD control toolkit. }\end{array}$ \\
\hline
\end{tabular}

goal is to reduce the premature morbidity and mortality from RF/RHD. Efforts were made to design a comprehensive RHD control program that included components of primary, secondary and tertiary prophylaxis (figure 2). The overall responsibility of managing the program was taken by NHF with the program director as a key functioning person (figure 3). Collaborating health facilities were selected that included governmental as well as non-governmental health facilities (figure 4).

Table 2 : Elements of the program

\begin{tabular}{|l|l|}
\hline 1. & Epidemiological Studies \\
\hline 2. & Awareness activities \\
\hline 3. & Training of health workers \\
\hline 4. & Case detection (Heart screening) \\
\hline 5. & Registry of RF/RHD patients \\
\hline 6. & $\begin{array}{l}\text { Delivery of medicines for secondary } \\
\text { prophylaxis }\end{array}$ \\
\hline 7. & Surveillance system \\
\hline 8. & Evaluation and monitoring \\
\hline
\end{tabular}

Figure 2 : Framework of the Nepalese Model of RF/RHD Control Program

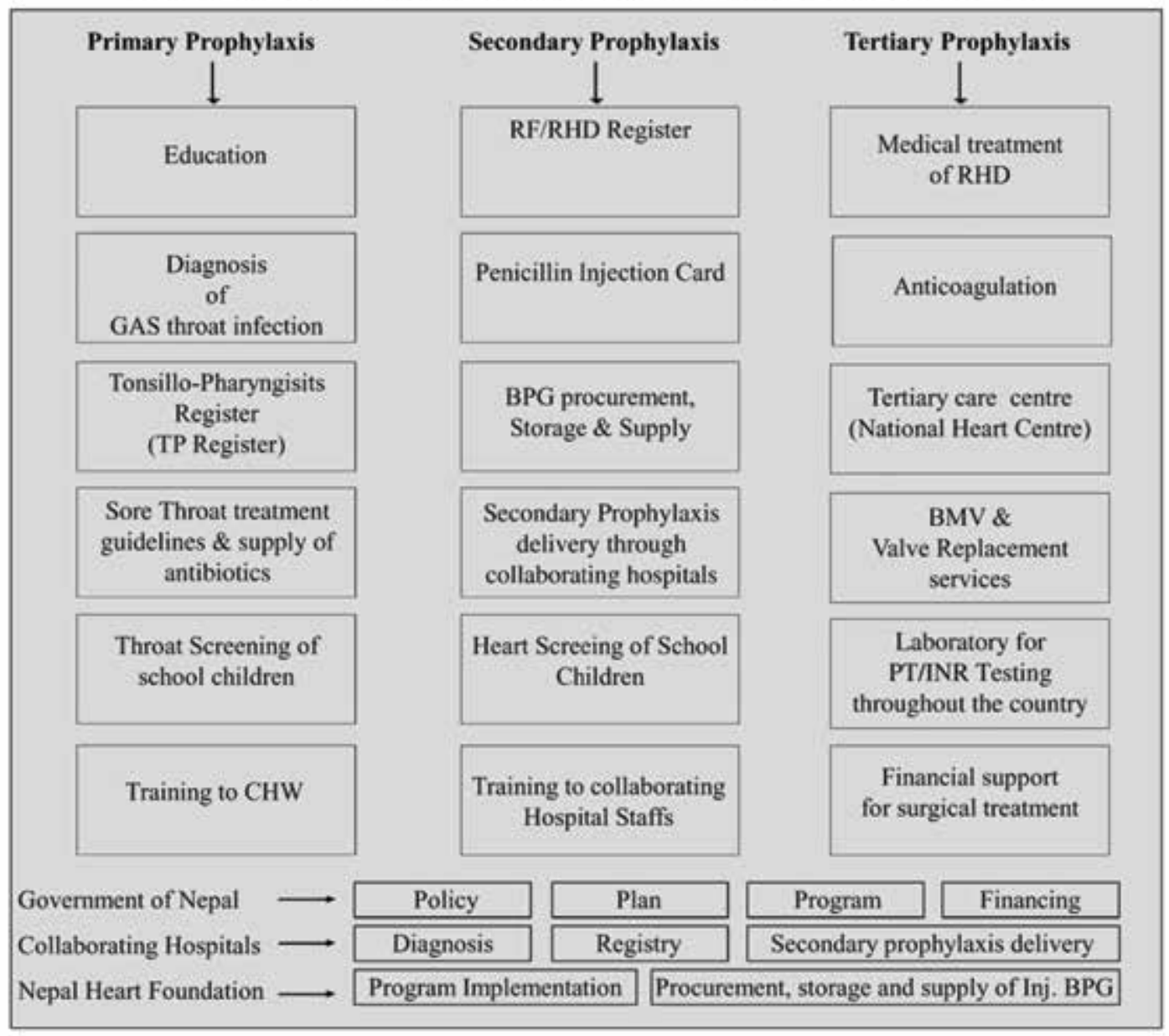


Figure 3 : Organogram of Nepalese RHD Control Program

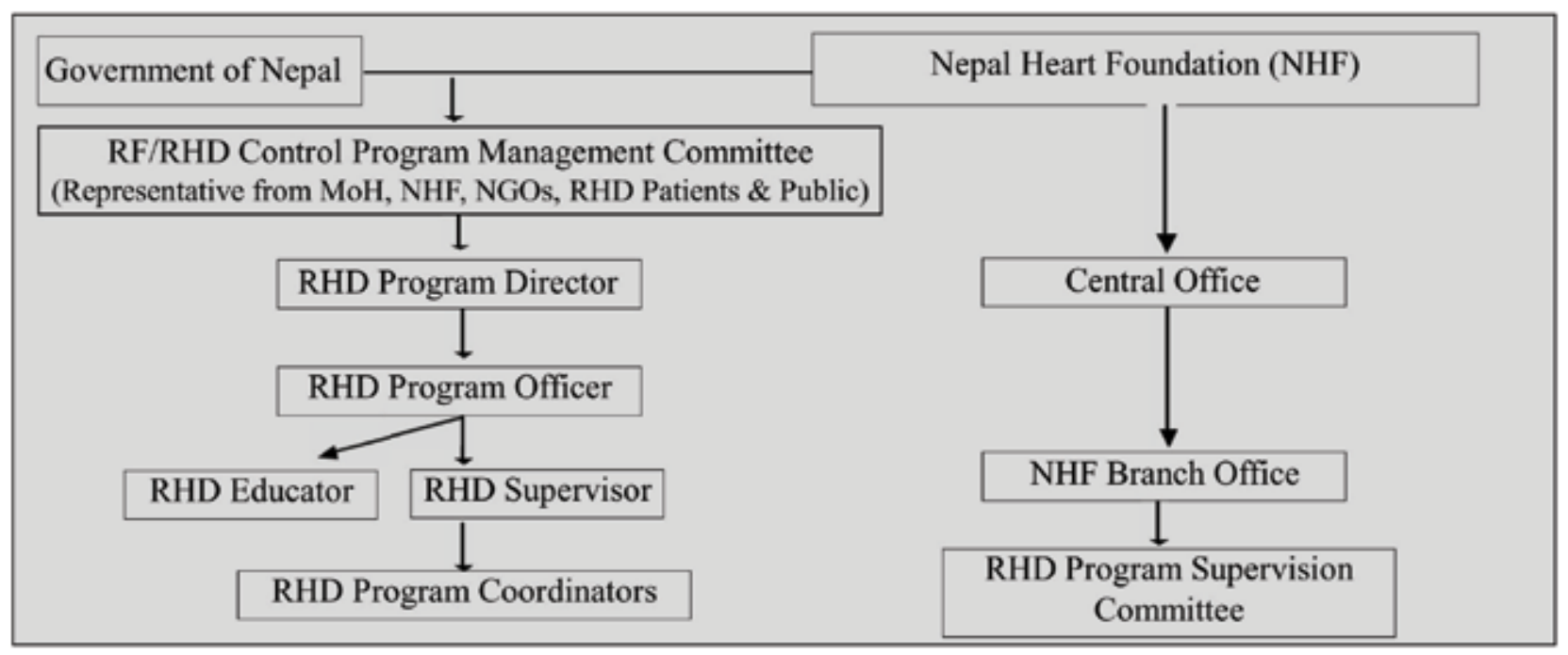

Figure 4 : Collaborating Health Facilities

\begin{tabular}{|c|c|}
\hline National Heart Centre \\
\hline \multicolumn{1}{|c|}{ Central Hospitals } \\
\hline Regional Hospitals \\
\hline Zonal Hospitals & Medical Colleges \\
\hline District Hospitals & Community Hospitals \\
\hline Primary Health Centre & Community Heart Clinics \\
\hline
\end{tabular}

RF/RHD Registers and the Penicillin Injection Card The RHD control program in Nepal maintains registers as a core component of the disease control efforts ${ }^{24}$. Nepal has adopted a three tiered system for maintaining the RF/RHD registry:

\section{National register}

All patients receiving Injection BPG for secondary prophylaxis nationwide are entered into the national (Central) RF/RHD register which is maintained at the program office of NHF. This is a computer register.

\section{Hospital register}

This paper register is maintained at all the hospitals and health facilities which participate in the RHD control program. Initially 22 government hospitals participated in the program, but by the end of April 2016, 38 health facilities (Central, Regional, Subregional, Zonal, few district \& community hospitals, medical colleges) are delivering secondary prophylaxis and maintain RF/RHD register with details of RF/RHD patients. These hospitals forward the data to the national register.

\section{Penicillin Injection Card}

A penicillin injection card is issued to all the patients receiving secondary prophylaxis. This card contains patient's information, diagnosis, batch number and brand name of the BPG injection that the patient is receiving, dates of injections given, due date and signature of health personnel delivering the injection.

\section{Advocacy, Awareness and Training}

NHF has conducted a range of activities to promote advocacy, awareness and training for RHD control. NHF has been leading the advocacy campaign along with the Nepal Heart Network (a network of 19 heart related organizations) and Nepal Non communicable Disease alliance to strengthen the engagement of GoN in RHD prevention and control. Attempts are continued to include RHD into Nepal NCD action plan. As a result RHD has been included into the school curriculum, valve surgery is made free for children below 15 years age, Percutaneous Transvenous Mitral Commissurotomy (PTMC) procedure is made free for all RHD patients, secondary prophylaxis is available free of cost throughout the country and medicines for primary prophylaxis is free through all primary health care facilities in the country. ${ }^{24}$

Community awareness activities are essential for a successful RHD control program. Health Literacy at baseline in Nepal has been limited; few school children, parents or teachers were aware that untreated streptococcal throat infection could lead to RHD. ${ }^{25}$ NHF has conducted several activities to increase awareness about RHD. The activities include distributing pamphlets, calendars, and posters, putting large hoarding boards throughout the country, telecast of documentary film on RHD on national TV, radio jingles on FM radios, public interaction programs on RHD and many others.

Training of health workers is another important component of comprehensive RHD control program. NHF has provided training to more than 1500 community health workers on RF and RHD. Orientation training for paramedics and hospital staffs participating in the secondary prophylaxis program is provided to all. 26 medical persons have received training of trainers (TOT) on RF and RHD. 
Practical issues in secondary prophylaxis delivery Secondary prophylaxis of RHD requires regular administration of long acting penicillin injection-Injection BPG (Benzathine Penicillin G), to prevent recurrent GAS infections and RF in patients with a history of RF or RHD. Nepal follows three weekly regimen of secondary prophylaxis as recommended by WHO. An alternative method is the use of oral Phenoxymethyl Penicillin tablet. Although less effective then injection BPG, it is used in those cases when Inj. BPG administration is not possible. NHF has been discouraging the use of oral penicillin citing that even with optimal patient adherence, the risk of recurrences is higher in individuals receiving oral prophylaxis than in those receiving intramuscular BPG. ${ }^{26}$

A number of challenges have to be faced on initiating and implementing RHD control program in low resource settings. Few practical issues that were faced and addressed by NHF were:

\section{Penicillin allergy and penicillin skin testing recommendations.}

Allergic reactions to penicillin are rare. The long term benefits of BPG therapy in preventing RF far outweigh the risk of serious allergic reaction. ${ }^{27}$ Patients and health care providers are very much concerned about the risk of anaphylactic reaction from BPG. ${ }^{28}$ Paramedics in Nepal expressed their serious concern about community reaction following fatal anaphylactic reaction. They suffered physical assault, claims for financial compensation and even jail sentence in some cases. This resulted in refusal by paramedics to administer Inj. BPG. In India some states even banned Inj. BPG. ${ }^{29}$ There were concerns about frequency of penicillin skin testing. Due to lack of published recommendations from international authorities paramedics and hospitals in Nepal were in confusion whether penicillin skin testing was to be done routinely before every penicillin injection. The practice varied from routine penicillin skin testing in one hospital to not testing at all in another hospital. NHF conducted several trainings to paramedics to overcome these concerns. They were provided training on safety measures in BPG delivery, anaphylaxis management and penicillin skin testing. NHF developed recommendations on penicillin skin testing (Table 3) and safe penicillin injection delivery (Table 4). The recommendation on penicillin skin testing was based on the audit of adverse reaction of 77300 penicillin injections delivered to 4713 RHD patients. ${ }^{30}$

Table 3 : NHF recommendations on penicillin skin testing

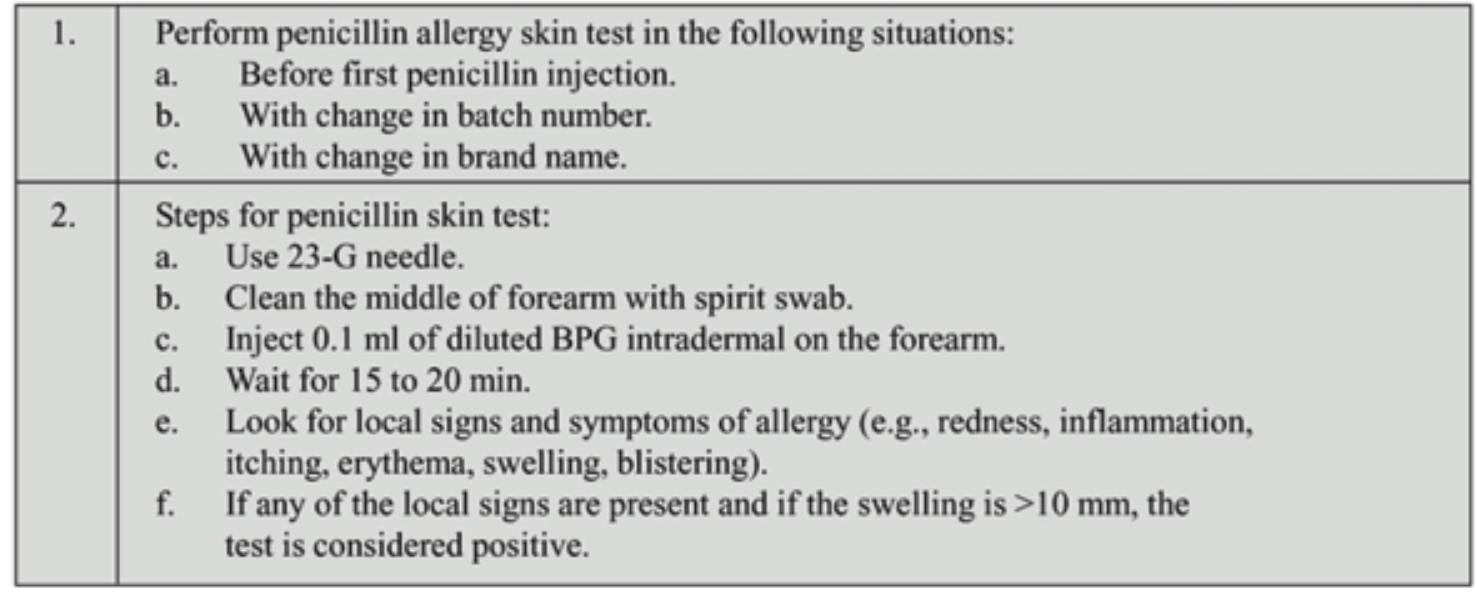

BPG Benzathine penicillin $G$

Table 4 : NHF recommendations on Safe BPG delivery

\begin{tabular}{|c|c|}
\hline 1. & Take consent from the patient or his/her relative before the first penicillin injection. \\
\hline 2. & Record the brand name and batch number of the BPG. \\
\hline 3. & Reconstitute the BPG powder with $3.5 \mathrm{ml}$ of sterile distilled water. \\
\hline 4. & Use 2 separate needles: 1 for pricking the vial and the other for injecting into the patient. \\
\hline 5. & Use $10 \mathrm{ml}$ syringe and $21-\mathrm{G}$ needle for deep intramuscular injection. \\
\hline 6. & $\begin{array}{l}\text { Patient should lie down on trolley or bed on abdomen with head resting on pillow in a } \\
\text { comfortable and relaxed position. In hospital settings, bed should be portable to rush the patient } \\
\text { to the intensive care unit in case of emergency. }\end{array}$ \\
\hline 7. & Inject BPG deep intramuscularly in the upper outer quadrant of the buttock. \\
\hline 8. & $\begin{array}{l}\text { Stay prepared for the treatment of possible anaphylaxis. } \\
\text { The following medicines and instruments should be ready for emergency use: } \\
\text { a. Adrenaline injection: I ampoule pre-loaded into the syringe. } \\
\text { b. Atropine injection. } \\
\text { c. Dexamethasone or antihistamine injection. } \\
\text { d. Intubation set. } \\
\text { e. Suction machine. }\end{array}$ \\
\hline
\end{tabular}




\section{BPG: Quality, stock and supply}

BPG is not manufactured in Nepal. It has to be imported from India. There are two main quality concerns about the imported powdered formulation of BPG. The first concern is difficulty in dissolving the powder which causes blockage in the needle during delivery, also increases pain and inaccurate dosing. The second concern is the duration of serum penicillin concentration levels. Poor quality formulation is difficult to dissolve, inject and concentration of penicillin falls more quickly than expected. ${ }^{31,32} \mathrm{NHF}$ while procuring the BPG for the RHD control program is strongly concerned to import the best quality BPG formulation.

Another important issue has been concerns about uninterrupted supply of BPG. Stock outs and shortages of BPG have occurred in Nepal in 2010 and 2015. The undeclared economic blockade at southern Nepalese border resulted is shortage of BPG in 2015. Although NHF keeps stock of BPG for 6 months, the stock went out and alternative method with oral penicillin was temporarily recommended by the NHF.

\section{Safe penicillin injection delivery and the penicillin injection room}

One of the objectives of the Nepalese RHD control program was to establish centers for safe delivery of inj. BPG. Hospitals with large numbers of patients receiving secondary prophylaxis were advised to have a separate room dedicated only for penicillin injection delivery. Hospitals with smaller number of RHD patients could use the same room for injection and dressings. The RHD control program had to put tremendous efforts to establish injection delivery rooms for safe and smooth injection administration and managing fear and anxiety for paramedics and for patients. NHF developed recommendations to standardize the process, maximize safety and minimize the risks (Table 4). Staffs were trained to diagnose and treat anaphylaxis. Rooms were equipped with an emergency care kit box distributed to all participating secondary prophylaxis centers by NHF. This box contained instruments and medicines to manage patients with anaphylaxis. Recommendations were made for a model penicillin injection delivery room to include patient trolley, oxygen cylinder, IV stand, suction machine, Intubation set and emergency care kit with necessary medicines.

\section{Pain from penicillin injection and dropouts.}

Although there is conflicting evidence about how much pain from injections impacts on patient adherence, it is reasonable to seek to minimize discomfort. ${ }^{33}$ It has been reported that $4.9 \%$ of the RHD patients drop out from secondary prophylaxis in Nepal due to injection phobia caused by pain. NHF continues to provide training to the health providers and has come up with recommendations to minimize pain during Inj. BPG administration: use of 21 gauze needle, warm room temperature, applying finger pressure at injection site, pushing the suspension slowly with constant pressure, patients distraction etc. Some programs mix BPG with local anesthetic to reduce the pain. There is reasonable evidence that using local anesthetic reduces pain without compromising serum concentration of BPG. ${ }^{34,35}$ However, this practice is not currently licensed, nor supported by BPG manufacturers.

\section{Practical issues in primary prophylaxis delivery}

Pharyngitis is a common childhood illness with up to $26 \%$ of these infections being caused by GAS. ARF is thought to occur in $0.3 \%$ to $3 \%$ of individuals infected with GAS as an autoimmune response to the initial infection. Primary prophylaxis strategies focus on the early diagnosis and timely treatment of GAS pharyngitis with antibiotics to prevent the autoimmune consequences resulting from the infection susceptible individuals. It is thought that antibiotic therapy initiated within 9 days of onset of pharyngitis is effective in preventing ARF. ${ }^{36}$ Significant barrier to the adequate diagnosis and treatment of GAS pharyngitis and thus primary prevention remain namely: 1) the diagnosis of GAS pharyngitis 2) treatment options 3 ) patient and physician awareness and 4) the positioning of primary prevention within a control program.

RHD control program in Nepal has initiated primary prophylaxis intervention as a pilot project in Lalitpur district since 2014. NHF is collaborating with district public health office, Lalitpur and Rotary Club of Patan, Rotary International district 3292 to launch this pilot project. Lalitpur district has a population of 400000 of which $40 \%$ are children of 5-16 years of age. There are 42 primary health care facilities participating in this program. Some of the practical issues needed to be addressed on implementing the primary prevention program were as follows:

\section{Tonsillo-Pharyngitis Register (TP Register)}

Register for secondary prophylaxis of RHD has been recommended by WHO and WHF. There is no mention about registers for primary prophylaxis. RHD control program in Nepal has established registers for primary prophylaxis also. All primary health care facilities participating in the primary prophylaxis program maintains TP Register. Details of children with streptococcal sore throat receiving treatment with antibiotic are entered into the paper register. They are followed up for evaluating result of treatment and recurrence of throat infection. Data from all the primary health care facilities are sent to the district public health office and program office at NHF. The advantage of maintaining TP Registers will be evaluated at the end of the project.

\section{Diagnosis of streptococcal tonsillitis and pharyngitis}

Developing a protocol for diagnosis of GAS tonsillitis and pharyngitis has been challenging. The gold standard confirmatory test for GAS pharyngitis is largely accepted to be a throat swab culture that is positive result for GAS. ${ }^{37}$ But this method is financially not viable in Nepal. NHF recommends to make diagnosis based on clinical signs and symptoms (throat pain, high fever, enlarged tonsils with exudates and pus spots, enlarged anterior cervical lymph nodes, absence of cough and running nose) aiming for a financially viable "treat all" approach. ${ }^{38}$ Program recommends treating not only clinically clear cut but also doubtful GAS pharyngitis cases.

\section{Oral antibiotic versus intramuscular Injection BPG}

Treatment of streptococcal throat infection in children with injection BPG was never practiced in Nepal. This was due to the fear of adverse reaction to penicillin. Good alternatives to Inj. BPG are oral Penicillin, Amoxicillin and Azithromycin. The latter two of these oral antibiotics are included in the essential drugs list by the GoN and are supplied free of cost to the rural people though primary health care facilities. RHD control program in Nepal has been evaluating the efficacy of oral Azithromycin in once daily for 5 days dosing. It is believed that the compliance to treatment with Azithromycin is better than with Amoxicillin or oral penicillin $V$ due to convenient once daily dosing and comparatively short duration of treatment.

\section{Midterm outcomes and the impact}

The secondary prevention program has completed 8 years of implementation. The evaluation of the impact on RHD control was done recently by NHF. The RHD burden trend was studied with the evaluation of three indicators. First indicator was the prevalence of RHD in School children of Kathmandu which came down from 1.2 per 1000 in 2003 to 0.8 in 2013.,.10 (Figure 5). Second indicator was the number of admissions for RHD in SGNHC where majority of 
the RHD patients are being referred. Among all medical admissions there were $14.5 \%$ RHD cases in 2011 against only $6.0 \%$ in the year $2015 .{ }^{39}$ The third indicator was the number of interventional procedures performed for RHD. Among all interventions performed for RHD in SGNHC, PTMC was performed in 400 patients in 2009 against 422 in 2015 and valve surgeries were performed in $36 \%$ cases in 2009 against $35 \%$ in $2015 .{ }^{39}$

It was concluded that the secondary prevention program has been effective in reducing the burden as well as the severity of RHD cases in Nepal, but the case load of interventional procedures for RHD still remains high. The report will be published soon.

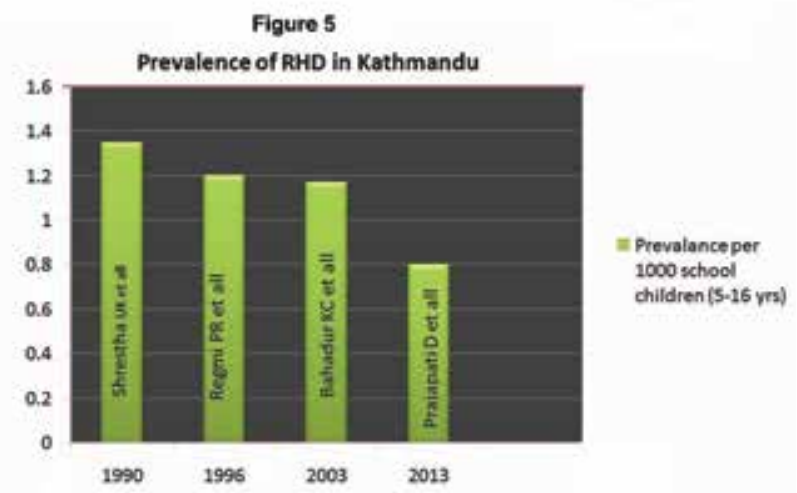

\section{Conclusions}

Tremendous efforts have been made in Nepal for the prevention and control of RF and RHD. The Nepalese model of RHD control program is an example of comprehensive and diagonal approach to RHD control in low resource countries. The government of Nepal has been supportive in the implementation of the program. The extensive involvement of NHF in coordinating, implementing and monitoring has been critical for success. The long term impact of the program needs to be evaluated but hopefully will end with good results.

\section{References}

1. Carapetis JR, Steer AC, Mulholland ER, Weber M. The global burden of group A Streptococcal diseases. Lancet infect Dis 2005;5:685-94.

2. Shrestha BR, Baniya GB, Raut KB, Sharma S. Rheumatic Fever in adults. J Nepal Med Assoc 1996;34:236-41

3. Shrestha UK, Bhattarai TN, Pandey MR. Prevalence of Rheumatic fever and Rheumatic Heart Disease in school children in a rural community of the hill region of Nepal. Indian Heart J 1991; 43: 39-41

4. Regmi PR, Pandey MR. Prevalence of Rheumatic Fever and Rheumatic Heart Disease in school children of Kathmandu city. Indian Heart J 1997; 49: 518-20

5. Bahadur KC, Sharma D, Shrestha MP, et. Al. Prevalence of Rheumatic and Congenital Heart Disease in school children of Kathmandu Valley in Nepal. Indian Heart J 2003; 55: 61518

6. Shrestha NR, Karki P, Mahato R, Gurung K, Pandey N, Agrawal K, Rothenbuhler M, Urban P, Juni P, Pilgrim T. Prevalence of Subclinical Rheumatic Heart Disease in Eastern Nepal.JAMA Cardiol. 2016; 1 (1): 89-96

7. World Health Organization. The WHO global programme for the prevention of Rheumatic Fever and Rheumatic Heart Disease: Report of a consultation to review progress and develop future activities. Geneva, Switzerland: WHO; 2000
8. World Health Organization study group. Rheumatic Fever and Rheumatic Heart Disease report of a WHO study group. Geneva, Switzerland: WHO; 1988

9. Nordet P, Lopez R, Duenas A, Sarmiento L. Prevention and control of rheumatic fever and rheumatic heart disease: the Cuban experience (1986-1996-2002). Cardiovasc J Atr 2008;19:135-40

10. Prajapati D, Sharma D, Regmi PR, Khanal H, Baidya SG, Rajbhandari S et al. Epidemiological survey of Rheumatic Fever, Rheumatic Heart Disease and Congenital Heart Disease among school children in Kathmandu Valley of Nepal. Nepalese Heart J 2013; 10:1-5

11. Remenyi B, Carapetis J, Wyber R , Taubert K, Mayosi B. Position statement of World Heart Federation on the prevention and control of Rheumatic Heart Disease. Nature Reviews Cardiology 2013; 10:284-92

12. Argueda A, Mohs E. Prevention of Rheumatic Fever in Costa Rica. J Pediatr 1992; 121: 565-72

13. Bach F, Chalons S, Forier E, et al. 10 years educational programme aimed at rheumatic fever in two French Caribbean Islands. Lancet 1996; 347: 644-8

14. McDonald M, Brown A, Noonan S, Carapetis JR. Preventing recurrent rheumatic fever: The role of register based programs. Heart 2005; 91:1131-3

15. World Health Organization. Community Prevention and control of Cardiovascular Disease. Geneva, Switzerland: WHO; 1986

16. WHO Rheumatic Fever and Rheumatic Heart Disease. WHO Technical Report Series. Geneva Switzerland: WHO; 2001

17. World Heart Federation. Diagnosis and Management of Acute Rheumatic Fever and Rheumatic Heart Disease. Geneva, Switzerland: World Heart Federation; 2008

18. Carapetis JR, Rheumatic Heart Disease in developing countries. N Engl J Med 2007; 357:439-41

19. Marijon E, Mirabel M, Celermajer DS, Jouven X. Rheumatic Heart Disease. Lancet 2012; 379:953-64

20. Wyber R. A conceptual framework for comprehensive rheumatic heart disease control programs. Global Heart 2013; 8(3): 241-6

21. Wyber R, Grainger Gasser A, Thompson D, Kennedy D, Johnson T, Taubert K, Carapetis J. Tools for implementing RHD control programmes (TIPs) Handbook. World Heart Federation and RhEACH. Perth, Australia 2014

22. Limbu Y, Maskey A, Current status of rheumatic fever and rheumatic heart disease in Nepal.J Nepal Medical Assoc 2002; 41: 514-7

23. Frenk J. bridging the Divide: Comprehensive reform to improve health in Mexico. Nairobi, Kenya: commission on Social Determinants of health; 2009

24. Regmi P, Upadhyaya A, Rheumatic Fever and Rheumatic Heart Disease prevention and control program in Nepal. Nepalese Heart J 209; 6:88-93

25. Regmi P. proceedings of "Have a Heart, Save a Heart" project. South Asian Youth Summit (SAYS) 2011 with support from the US Embassy and technical support from the Nepal Heart Foundation: Kathmandu, Nepal: 2012

26. Feinstein AR, Wood HF, Epstein JA, Taranta A, Simpson R, Tursky E. A controlled study of three methods of prophylaxis against streptococcal infection in a population of rheumatic children III: results of the first three years of the study including methods for evaluating the maintaince of oral prophylaxis. $\mathrm{N}$ Engl J Med 1959; 260:689-702

27. World Health Organization. Rheumatic Fever and Rheumatic Heart Disease. Geneva, Switzerland: World Health Organization; 2004 
28. Padmavati S. Rheumatic Heart Disease: prevalence and preventive measures in Indian Subcontinent. Heart 2011; 80:127

29. Shah B, Sharma M, Kumar R, Brahmadathan K, Abraham V, Tandon R, Rheumatic heart disease: progress and challenges in India. Indian Journal of Pediatrics 2012; 80 (Supp): S77-S86

30. Regmi P, Upadhyaya A. Allergic reaction to long-term benzathine penicillin injection for secondary prevention of acute rheumatic fever and recommendations for skin testing. Nepalese Heart J 2011; 8:16-8

31. Wyber R, Taubert K, Marko S, Kaplan E. Benzathine Penicillin $\mathrm{G}$ for the management of RHD: concerns about quality and access, and opportunities for intervention and improve-ment. Global Heart 2013; 8(3): 227-34

32. Broderick M, Hansen C, Faix D. Factors associated with loss of penicillin $\mathrm{G}$ concentrations in serum after intramuscular benzathine penicillin $\mathrm{G}$ injection: a meta-analysis. Pediatric infections diseases Journal 2012; 31: 722-5

33. Tullu M, Ghandi A, Ghildiyal R. Benzathine Penicillin prophylaxis in children with rheumatic fever/rheumatic heart disease: a study of compliance. Al Ameen Journal of Medical Science 2010; 3(2): 140-5

34. Amir J, Ginat S, Cohen Y, Marcus T, Keller N, Versano I. Lidocaine as a diluent for administration of benzathine penicillin G. Pediatric Infections Disease Journal 1998; 17(10):
890-3

35. Morsy M, Mohamed M, Abosedira M, et al, Lidocaine as a dilutant for benzathine penicillin $G$ reduces injection pain in patients with rheumatic fever: a prospective, randomized, double-blinded crossover study. Australian Journal of Basic and Applied Science 2012; 6(5): 236-40

36. Gerber MA, Baltimore RS, Eaton CB, et al. Prevention of rheumatic fever and diagnosis and treatment of acute streptococcal pharyngitis: a scientific statement from the American Heart Association Rheumatic Fever, Endocarditis, and Kawasaki Disease Committee of the Council on Cardiovascular Disease in the Young, the Interdisciplinary Council on Functional Genomics and Translational Biology, and the Interdisciplinary Council on Quality of Care and Outcomes Research. Circulation 2009;119:1541-51.

37. Kaplan El. The group A streptococcal upper respiratory tract carrier state: an enigma.J Pediatr 1980; 97: 337-45

38. Irlan J, Mayosi B, Engel M, Gaziano TA. Primary prevention of acute rheumatic fever and rheumatic heart disease with penicillin in South African children with pharyngitis: a cost effectiveness analysis. CircCardiovascQual Outcomes 2013; 6:313-51

39. Shahid Gangalal National Heart Center, Annual report 20092015
Cite this article as: Prakash Raj Regmi. Comprehensive approach to Rheumatic Fever and Rheumatic Heart Disease prevention and control: The Nepalese Model Nepalese Heart Journal 2016;13(2): 3-10. 